\title{
Increased mortality of COVID-19 infected diabetes patients: role of furin proteases
}

\author{
Senthil Kumar Ganesan $\mathbb{1}^{1,2} \cdot$ Purushothaman Venkatratnam ${ }^{3} \cdot$ Jaideep Mahendra ${ }^{4} \cdot$ Nalini Devarajan $^{5}$
}

Received: 4 June 2020 / Revised: 6 August 2020 / Accepted: 19 August 2020 / Published online: 1 September 2020

(c) Springer Nature Limited 2020

\section{To the Editor:}

The entire world is currently under the big threat of coronavirus disease (COVID-19) caused by the Severe Acute Respiratory Syndrome Coronavirus 2 (SARS CoV2) virus. This pandemic disease has affected more than 6.5 million people globally till May 2020 and the death toll has crossed 380,000 . Epidemiological surveys show that diabetic patients are at high risk to contract COVID-19 infection [1]. More importantly, they are also the major victims of COVID-19 associated mortalities. Indeed, the mortality risk due to SARS CoV2 infection is fourfold higher in diabetic patients compared to nondiabetic individuals [2]. In a study done by the COVID-19 surveillance group of Italy on 3200 patients, $34 \%$ of 481 nonsurvivors had diabetes [3]. Likewise, in another study of 52 patients under intensive care, $22 \%$ of 32 nonsurvivors were diabetic [4]. Similarly, reports from the Centers for Diabetes Control and Prevention, United States show that $11 \%$ of COVID-19 patients have diabetes [5]. The most populated country like India, more

Senthil Kumar Ganesan

skumar@iicb.res.in

$\triangle$ Nalini Devarajan

nalinikrishu@gmail.com

1 Laboratory of Functional Genomics, Structural Biology \& Bioinformatics Division, CSIR-Indian Institute of Chemical Biology, TRUE Campus, CN Block-6, Sector V, Salt Lake, Kolkata 700091, India

2 Academy of Scientific and Innovative Research (AcSIR), Ghaziabad 201002, India

3 Meenakshi Ammal Dental College and Hospital, Maduravoyal, Chennai 600095 Tamil Nadu, India

4 Department of Periodontology, Meenakshi Ammal Dental College and Hospital, Maduravoyal, Chennai 600095 Tamil Nadu, India

5 Central Research Laboratory, Meenakshi Ammal Dental College, Meenakshi Academy of Higher Education and Research, Maduravoyal, Chennai, Tamil Nadu, India than $70 \%$ of COVID-19-associated mortalities are due to comorbidities including diabetes, hypertension, CVD, and pulmonary problems. The diabetic population has crossed 120 million worldwide in 2019 and this in turn poses an increased threat for further COVID-19 infection and related mortalities in these patients in upcoming days. Hence, understanding the pathobiology behind the increased mortality in COVID-19-affected diabetic patients is highly essential to develop prominent prognostic biomarkers and accurate therapies that save their lives.

One of the major proteins which is highly expressed in diabetic patients when compared to nondiabetic individuals and also a primary requisite for SARS CoV2 virus activation in host cells is furin. The Furin is a type 1 membranebound serine endoprotease enzyme that belongs to the subtilisin-like proprotein convertase family. Its major function is to activate the newly synthesized latent proteins by cleaving off the regions that render them biologically inactive. Accumulating in the Golgi apparatus, furin cleaves the precursor proteins just downstream of a basic amino acid target sequence (canonically, Arg-X-(Arg/Lys) -Arg') [6]. In diabetic patients, elevated plasma furin level acts as an independent predictor for disease onset, progression, and premature mortality [7]. Furthermore, it also provides information about CVD risk in these patients [8].

During SARS CoV2 virus infection, furin mediates viral entry into host target cells. After the binding of spike (S) glycoprotein of SARS CoV2 virus to angiotensinconverting enzyme 2 present in the host cell surface, cleavage (also called priming) of S1/S2 furin-like cleavage site in $\mathrm{S}$ protein is essential for the fusion of viral membrane with the host cell membrane. Furin cleaves this S1/S2 site and allows viral entry into host cells which in turn leads to the onset of COVID-19 infection in humans (Fig. 1) [9]. An important point to note is that $\mathrm{S} 1 / \mathrm{S} 2$ furin-like cleavage site is the unique feature present exclusively in $\mathrm{S}$ protein of SARS CoV2 virus, but absent in other SARS-CoV-related coronaviruses [10]. However, it is present in other pandemic viruses including human immuno virus, anthrax, ebola, and 
Marburg virus. Viruses with furin-like cleavage sites tend to be highly pathogenic in nature [10] and hence, it is more likely that the S1/S2 site in S protein of SARS CoV2 virus is responsible for its aberrant pathogenicity. Also, unlike other serine endoproteases whose expression is restricted to respiratory and gastrointestinal tracts, furin is widely distributed throughout the body with increased enrichment in lungs, kidneys, and endocrine organs, moderate expression in the digestive and gastrointestinal tract and low-level expression in muscle tissues (https://www.proteinatlas.org/ ENSG00000140564-FURIN/tissue). This in turn broadens the cell tropism of the SARS CoV2 virus and may probably be the reason behind the multiple organ damages observed in COVID-19 patients. This is proven by the fact that organs severely affected by SARS CoV2 infection in

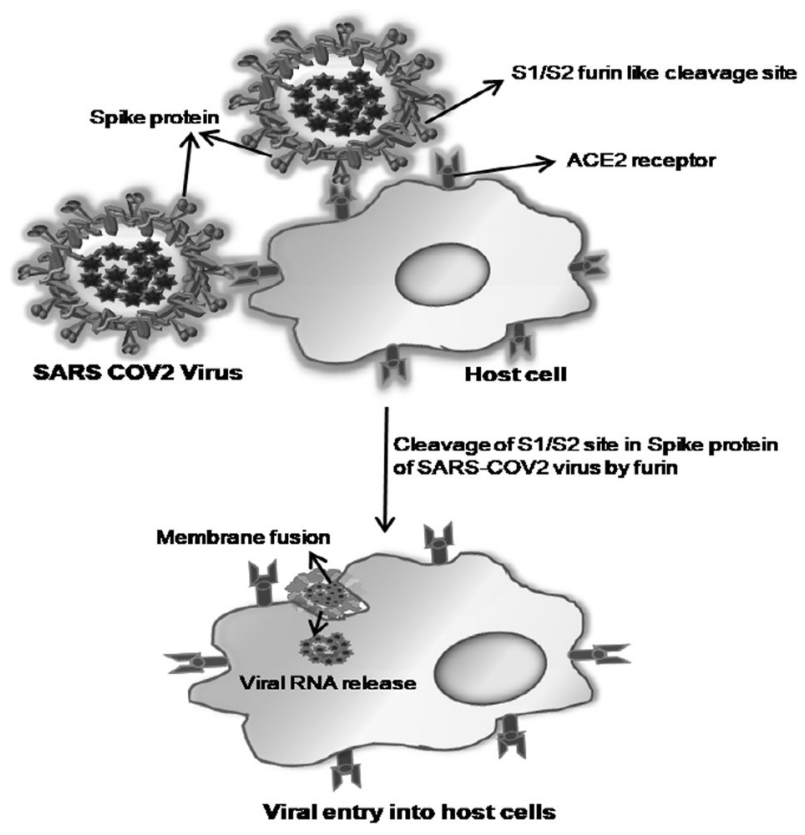

Fig. 1 Furin cleaves the S1/S2 furin-like cleavage site in "S" protein if SARS COV2 virus and mediates viral entry into host cells.
COVID-19 patients including lungs, liver, kidney express high levels of furin.

It is now obvious that furin plays a key role in the high pathogenicity, host cell entry, and wide cell tropism of the SARS CoV2 virus. Hence, furin overexpression in diabetes patients may be the key reason behind their increased risk to contract COVID-19 infection. Furthermore, due to poor glycemic control, diabetes patients show weak immune response against SARS CoV2 infection [11], and this in turn might favor augmented furin-mediated entry of the SARS CoV2 virus into target cells, leading to increased viral load. This might also potentiate viral secondary infections in the lungs, leading to acute respiratory distress syndrome. More importantly, furin is present abundantly in kidneys (https://www.proteinatlas.org/ENSG00000140564FURIN/tissue) and atherosclerotic plaques [12]. Hence, in patients with late diabetic complications including diabetic renal disease and cardiovascular disease, this endoprotease might induce severe viral attack in kidneys and atherosclerotic plaques, which in turn might trigger aggressive inflammatory storm and subsequent pathological events in these sites all of which eventually culminate in acute renal failure and/ or acute cardiac injury with heart failure. Blood clots are other major fatal complications that cause increased mortalities in COVID-19 patients [13]. Intriguingly, furin is involved in blood coagulation as well. This endoprotease cleaves and activates various precursor proteins including factor VII, factor IX, protein C, protein S, and Von Willebrand factors that are critically needed for blood hemostasis [14, 15]. Hence, furin might also be associated with COVID-19-related mortalities that occur due to blood clots.

In conclusion, furin, the primary requisite for SARS CoV2 virus entry into host cells is present in higher concentrations in the plasma of diabetic patients. This in turn might increase the susceptibility of diabetes patients to contract COVID-19 infection. Furthermore, the weak immune response in diabetes patients might allow increased
Fig. 2 Probable mechanism relating furin to increased mortality of COVID-19 infected diabetes patients.

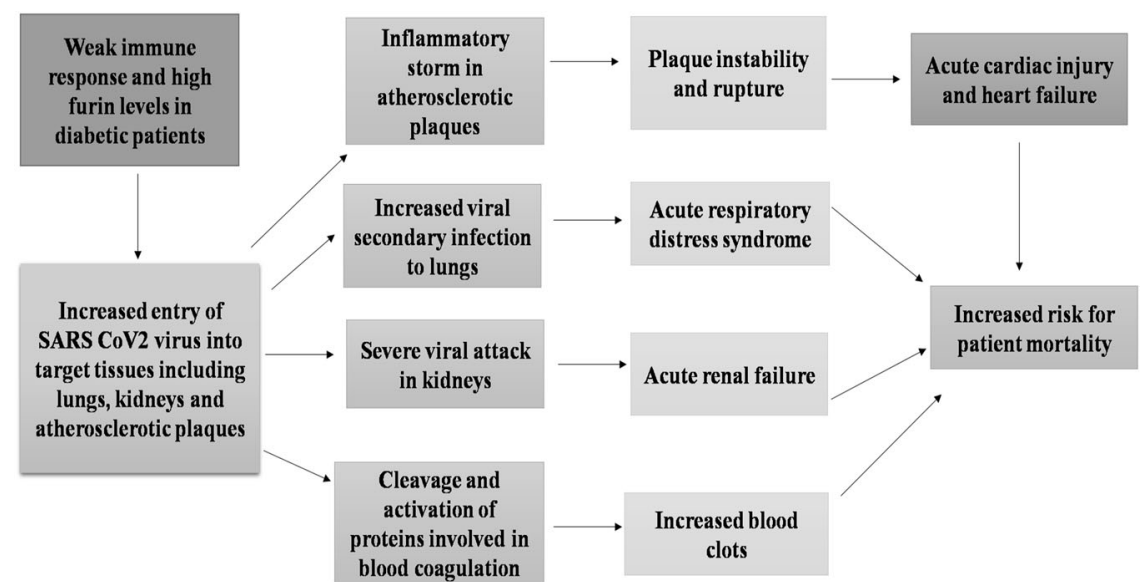


furin mediated viral entry in the target tissues, leading to enhanced viral load. This in turn might favor severe viral attack in sites that harbor more furin including lungs, kidney, and atherosclerotic plaques, leading to fatal complications including acute respiratory distress syndrome, acute renal failure, and acute cardiac injury with heart failure, respectively (Fig. 2). Hence, it is highly plausible that furin might be the major risk factor for the increased mortality of COVID-19 infected diabetes patients. Detailed investigations on the correlation between plasma furin levels, viral load, and severity of fatal complications in COVID-19infected diabetes patients are very much needed to decipher the link between furin and prognosis status in these patients. These findings will aid in determining if plasma furin could act as a prominent prognostic biomarker in COVID-19 patients. Also, it will provide clues for the utilization of furin inhibitors to save the lives of COVID-19-infected diabetes patients.

Acknowledgements The authors gratitude to all the medical professionals and scientists for their significant contribution to combat COVID19 pandemic.

Author contributions GSK, VP, JM, and DN have equally contributed to framing this commentary, collecting study materials, preparing a manuscript, and proofreading work.

\section{Compliance with ethical standards}

Conflict of interest The authors declare that they have no conflict of interest.

Publisher's note Springer Nature remains neutral with regard to jurisdictional claims in published maps and institutional affiliations.

\section{References}

1. Singh AK, Gupta R, Ghosh A, Misra A. Diabetes in COVID-19: Prevalence, pathophysiology, prognosis and practical considerations. Diabetes Metab Syndr. 2020;14:303-10.
2. Bloomgarden ZT. Diabetes and COVID-19. J Diabetes. 2020;12:347-8.

3. Onder G, Rezza G, Brusaferro S. Case-fatality rate and characteristics of patients dying in relation to COVID-19 in Italy. JAMA. 2020;323:1775-6.

4. Yang X, Yu Y, Xu J, Shu H, Xia J, Liu H, et al. Clinical course and outcomes of critically ill patients with SARS-CoV-2 pneumonia in Wuhan, China: a single-centered, retrospective, observational study. Lancet Respiratory Med. 2020;8:475-81.

5. CDC COVID-19 Response Team. Preliminary Estimates of the Prevalence of Selected Underlying Health Conditions Among Patients with Coronavirus Disease 2019 - United States, February 12-March 28, 2020. MMWR Morb Mortal Wkly Rep. 2020;69:382-6.

6. Thomas G. Furin at the cutting edge: from protein traffic to embryogenesis and disease. Nat Rev Mol Cell Biol. 2002;3:753-66.

7. Fernandez C, Rysa J, Almgren P, Nilsson J, Engstrom G, OrhoMelander $\mathrm{M}$, et al. Plasma levels of the proprotein convertase furin and incidence of diabetes and mortality. J Intern Med. 2018;284:377-87.

8. Fathy SA, Abdel Hamid FF, Zabut BM, Jamee AF, Ali MA, Abu Mustafa AM. Diagnostic utility of BNP, corin and furin as biomarkers for cardiovascular complications in type 2 diabetes mellitus patients. Biomarkers. 2015;20:460-9.

9. Hoffmann M, Kleine-Weber H, Pöhlmann S. A multibasic cleavage site in the spike protein of SARS-CoV-2 is essential for infection of human lung cells. Mol Cell. 2020;78:779-84.e5.

10. Coutard B, Valle C, de Lamballerie X, Canard B, Seidah NG, Decroly E. The spike glycoprotein of the new coronavirus 2019nCoV contains a furin-like cleavage site absent in $\mathrm{CoV}$ of the same clade. Antiviral Res. 2020;176:104742.

11. Critchley JA, Carey IM, Harris T, DeWilde S, Hosking FJ, Cook DG. Glycemic Control and Risk of Infections Among People With Type 1 or Type 2 Diabetes in a Large Primary Care Cohort Study. Diabetes Care. 2018;41:2127-35.

12. Ren K, Jiang T, Zheng XL, Zhao GJ. Proprotein convertase furin/ PCSK3 and atherosclerosis: New insights and potential therapeutic targets. Atherosclerosis. 2017;262:163-70.

13. Willyard C. Coronavirus blood-clot mystery intensifies. Nature. 2020;581:250.

14. Wasley LC, Rehemtulla A, Bristol J, Kaufman R. PACE/furin can process the vitamin K-dependent pro-factor IX precursor within the secretory pathway. J Biol Chem. 1993;268:8458-65.

15. Bendetowicz AV, Morris JA, Wise RJ, Gilbert GE, Kaufman RJ. Binding of factor VIII to von Willebrand factor is enabled by cleavage of the von Willebrand factor propeptide and enhanced by formation of disulfide-linked multimers. Blood. 1998;92:529-38. 